\title{
Ambiguities on the quantization of a one-dimensional dissipative system with position depending dissipative coefficient
}

\author{
G. López ${ }^{1}$, X. E. López ${ }^{2}$ and G. González ${ }^{3}$ \\ ${ }^{1}$ Departamento de Física de la Universidad de Guadalajara \\ Apartado Postal 4-137 \\ 44410 Guadalajara, Jalisco, México \\ ${ }^{2}$ Facultad de Ciencias de la UNAM \\ Apartado postal 70-348, Coyoacán 04511 México D.F. \\ ${ }^{3}$ Departamento de Matemáticas y Física, ITESO \\ Periférico Sur 8585, C.P. 45090 \\ Guadalajara, Jalisco, México \\ PACS: 03.20.+i, 03.30.+p, 03.65.-w
}

May, 2005

\begin{abstract}
For a one-dimensional dissipative system with position depending coefficient, two constant of motion are deduce. These constants of motion bring about two Hamiltonians to describe the dynamics of same classical system. However, their quantization describe the dynamics of two completely different quantum systems.
\end{abstract}




\section{Introduction}

It is well known that the Lagrangian (therefore, the Hamiltonian) formulation for some systems of more than one dimensions may not exist [1]. Fortunately, many of our physical systems have avoided this problem and the whole quantum and statistical mechanics of nondissipative systems are given in terms of the Hamiltonian or Lagrangian formulation. For dissipative systems there are two main approaches, the first one consists on keeping the same Hamiltonian formalism for the system and the interacting background. By doing this, one brings about a master equation, and the dissipation and diffusion parameters appear as a part of the solution [2]. This approach has its own merits, but it will not be fallowed on this paper. We will follow the second approach which consists in obtaining a phenomenological velocity depending Hamiltonian which represents the classical dissipative system and proceeding to make the usual quantization with this Hamiltonian. Within this approach, one can additionally study the mathematical consistence of the Hamiltonian quantum mechanics. Now, even for one-dimensional systems, where the existence of their Lagrangians is guaranteed [3], the Lagrangian and Hamiltonian formulation are not free of problems [4]. One of the problems we are concerned is the implication on the quantization of a classical system when different Hamiltonians describe the same classical system [5]. This problem would represent an ambiguity on the quantum mechanics for the quantization of a classical system. To study this problem, we consider a one-dimensional velocity depending force which may represent a dissipative system with proper selection of parameters. Following the procedure given in reference [6], two constant of motion with energy units are found for this system. Their associated Lagrangians and Hamiltonians are deduced, and, using the perturbative quantum theory, the two resulting quantum dynamics are shown with these Hamiltonian.

\section{Constants of Motion}

Let us consider a one-dimensional system described by the following equation

$$
m \frac{d^{2} x}{d t^{2}}=-U_{x}\left(1-\alpha \dot{x}^{2}\right)
$$

where $m$ is the mass of the particle, $x$ and $\dot{x}$ denote its position and velocity, $U_{x}$ represents the differentiation with respect to $x$ of the function $U(x)$, and $\alpha$ is a real parameter. This equation could represent the motion of a particle under a force $-U_{x}$ in a dissipative medium characterized by a quadratic velocity force (for $\dot{x}>0$ and $\alpha<0$ ) with a coefficient depending on the position of the form $\alpha U_{x}$. Eq. (1) can be written as the following autonomous dynamical system

$$
\frac{d x}{d t}=v \quad \frac{d v}{d t}=-U_{x}\left(1-\alpha v^{2}\right) / m
$$

which has the set $S_{c}=\left\{\left(x_{c}, 0\right) \in \Re^{2} \mid U_{x}\left(x_{c}\right)=0\right\}$ as the set of critical points in the phase space $(x, v)[7]$, which is the same as that for $\alpha=0$. A constant 
of motion for this system is a function $K=K(x, v)$ such that $d K / d t=0$, i.e., it satisfies the following partial differential equation [8]

$$
v \frac{\partial K}{\partial x}-\frac{U_{x}}{m}\left(1-\alpha v^{2}\right) \frac{\partial K}{\partial v}=0 .
$$

The general solution of this equation is given by [9]

$$
K_{\alpha}(x, v)=G(C)
$$

where $G$ is an arbitrary function of the characteristic curve of (3), denoted by $C$. This characteristic curve can by deduced as

$$
C=-\frac{m}{2 \alpha} \log \left(1-\alpha v^{2}\right)+U(x) .
$$

The function $G$ can be chosen such that in the limit for the dissipation parameter goes to zero, one can get the usual energy expression of a conservative system, $E=\lim _{\alpha \rightarrow 0} K_{\alpha}=m v^{2} / 2+U(x)$. There are at least two ways to do this. One way is by selecting $G$ as the identity function, $G(C)=C$, and the other is by selecting $G$ of the form $G(C)=e^{-2 \alpha C / m} / \alpha+m / 2 \alpha$. These two expressions for $G$ bring about the following constants of motion

$$
K_{\alpha}^{(1)}(x, v)=-\frac{m}{2 \alpha} \log \left(1-\alpha v^{2}\right)+U(x)
$$

and

$$
K_{\alpha}^{(2)}(x, v)=\frac{1}{2} m v^{2} e^{-2 \alpha U(x) / m}+\frac{m}{2 \alpha}\left(1-e^{-2 \alpha U(x) / m}\right) .
$$

\section{Lagrangian, Generalized Linear Momentum and Hamiltonian}

Using the known expression [10] to get the Lagrangian given the constant of motion,

$$
L(x, v)=v \int \frac{K(x, v) d v}{v^{2}},
$$

the Lagrangians associated to (6) and (7) are

$$
L_{\alpha}^{(1)}(x, v)=\frac{m v}{\sqrt{\alpha}} \operatorname{arctanh}(v \sqrt{\alpha})+\frac{m}{2 \alpha} \log \left(1-\alpha v^{2}\right)-U(x)
$$

and

$$
L_{\alpha}^{(2)}(x, v)=\frac{1}{2} m v^{2} e^{-2 \alpha U(x) / m}-\frac{m}{2 \alpha}\left(1-e^{-2 \alpha U(x) / m}\right) .
$$

Their generalized linear momenta $(p=\partial L / \partial v)$ are

$$
p_{\alpha}^{(1)}=\frac{m}{\sqrt{\alpha}} \operatorname{arctanh}(v \sqrt{\alpha})
$$

and

$$
p_{\alpha}^{(2)}=m v e^{-2 \alpha U(x) / m} .
$$


Thus, their associated Hamiltonians, $H_{\alpha}^{(1)}\left(x, p_{\alpha}^{(i)}\right)=K_{\alpha}^{(i)}\left(x, v\left(x, p_{\alpha}^{(i)}\right)\right)$, for $i=1,2$ are given by

$$
H_{\alpha}^{(1)}(x, p)=-\frac{m}{2 \alpha} \log \left(1-\tanh ^{2}\left(\frac{p \sqrt{\alpha}}{m}\right)\right)+U(x)
$$

and

$$
H_{\alpha}^{(2)}(x, p)=\frac{p^{2}}{2 m} e^{2 \alpha U(x) / m}+\frac{m}{2 \alpha}\left(1-e^{-2 \alpha U(x) / m}\right),
$$

where the same $p$ variable has been used for the generalized linear momentum to simplify the notation. Note from (9), (10), (11), (12), (13) and (14) that the following limits are satisfied

$$
\begin{gathered}
\lim _{\alpha \rightarrow 0} L_{\alpha}^{(i)}(x, v)=\frac{1}{2} m v^{2}-U(x), i=1,2 \\
\lim _{\alpha \rightarrow 0} p_{\alpha}^{(i)}=m v, i=1,2
\end{gathered}
$$

and

$$
\lim _{\alpha \rightarrow 0} H_{\alpha}^{(i)}(x, p)=\frac{p^{2}}{2 m}+U(x), i=1,2,
$$

corresponding to the conservative case of (1). At first order on the parameter $\alpha$, one gets from (13) and (14) the following Hamiltonians

$$
H_{1}=\frac{p^{2}}{2 m}+U(x)+\alpha \frac{19 p^{4}}{48 m^{3}}
$$

and

$$
H_{2}=\frac{p^{2}}{2 m}+U(x)+\alpha\left[\frac{p^{2} U(x)}{m^{2}}-\frac{2}{m} U^{2}(x)\right] .
$$

We must point out that the Lagrangians (9) and (10), the generalized momenta (11) and (12), and the Hamiltonians (13) and (14) are associated to the same system (1), that is, they generate the same classical dynamics.

\section{Quantization}

It is clear that Schödinger quantization of the system (1) using the corresponding Hermitian operator to the Hamiltonians (13) or (14),

$$
i \hbar \frac{\partial \Psi}{\partial t}=\hat{H}_{\alpha}^{(i)} \Psi
$$

will generate different quantum dynamics. Therefore, it is just necessary to show this difference at first order in the parameter $\alpha$ with the Hamiltonians (16) and (17) and at first order in perturbation theory [11]. Since (16) and (17) are time independent, one uses time independent perturbation theory. According to this theory, the corrections to the nth-eigenvalue and nth-eigenvector are given by

$$
E_{n}^{(i)}=E_{n}^{(0)}+\left\langle n\left|\widehat{W}_{i}\right| n\right\rangle, i=1,2
$$


and

$$
\psi_{n}^{(i)}(x)=\psi_{n}^{(0)}(x)+\sum_{k \neq n} \frac{\left\langle n\left|\widehat{W}_{i}\right| k\right\rangle}{E_{n}^{(0)}-E_{k}^{(0)}} \psi_{k}^{(0)}(x), i=1,2,
$$

where the Hermitian operators associated to the Hamiltonians (16) and (17) are written as

$$
\widehat{H}_{i}=\widehat{H}_{0}+\widehat{W}_{i}, i=1,2
$$

with $\widehat{H}_{0}$ and $\widehat{W}_{i}$ given by

$$
\begin{gathered}
\widehat{H}_{0}=\frac{\hat{p}^{2}}{2 m}+U(x), \\
\widehat{W}_{1}=\alpha \frac{19 \hat{p}^{4}}{48 m^{3}},
\end{gathered}
$$

and

$$
\widehat{W}_{2}=\alpha\left[\frac{p^{2} \widehat{U(x)}}{m^{2}}-\frac{2}{m} U^{2}(x)\right] .
$$

The energy $E_{n}^{(0)}$ and the wave function $\psi_{n}^{(0)}(x)$ are solutions of the eigenvalue problem

$$
\widehat{H}_{0} \psi_{n}^{(0)}(x)=E_{n}^{(0)} \psi_{n}^{(0)}(x) .
$$

One can also use the Dirac notation $\widehat{H}_{0}|n\rangle=E_{n}^{(0)}|n\rangle$, as it has been used in (19a) and (19b).

To get explicitly the values for (19a) and (19b), let us consider the harmonic oscillator potential $U(x)=m \omega^{2} x^{2} / m$, where $\omega$ is its natural frequency of oscillation. For this case, one can write (20) in terms of ascent and descent operators, $a^{+}$and $a$, as

$$
\widehat{H}_{0}=\hbar \omega\left(a^{+} a+\frac{1}{2}\right)
$$

where the operators $a^{+}$and $a$ have been defined as

$$
a^{+}=\sqrt{\frac{m \omega}{2 \hbar}} x-\frac{i}{\sqrt{2 m \hbar \omega}} \hat{p}
$$

and

$$
a=\sqrt{\frac{m \omega}{2 \hbar}} x+\frac{i}{\sqrt{2 m \hbar \omega}} \hat{p}
$$

which satisfy the following relations

$$
\left[a^{+}, a\right]=1, \quad a^{+}|n\rangle=\sqrt{n+1}|n+1\rangle, \quad a|n\rangle=\sqrt{n}|n-1\rangle, \quad a|0\rangle=0 .
$$

In addition, $E_{n}^{(0)}$ is given by

$$
E_{n}^{(0)}=\hbar \omega\left(n+\frac{1}{2}\right)
$$


Using Weyl idea, one has that the Hermitian operator associated to the product $p^{2} U(x)$ can be given by

$$
\widehat{p^{2}} \widehat{U(x)}=\frac{m \omega^{2}}{12}\left[x^{2} \hat{p}^{2}+\hat{p}^{2} x^{2}+x \hat{p} x \hat{p}+\hat{p} x \hat{p} x+x \hat{p}^{2} x+\hat{p} x^{2} \hat{p}\right] .
$$

Therefore, written $x$ and $\hat{p}$ in terms of $a^{+}$and $a$,

$$
x=\sqrt{\frac{\hbar}{2 m \omega}}\left(a^{+}+a\right), \quad \hat{p}=i \sqrt{\frac{m \hbar \omega}{2}}\left(a^{+}-a\right),
$$

and using (25a), one has

$$
\left\langle n\left|\widehat{W}_{1}\right| n\right\rangle=\alpha \frac{19 \hbar^{2} \omega^{2}}{192 m}\left(4 n^{2}+4 n+3\right)
$$

and

$$
\left\langle n\left|\widehat{W}_{2}\right| n\right\rangle=-\alpha \frac{\hbar^{2} \omega^{2}}{4 m}\left(2 n^{2}+2 n+1\right) .
$$

Thus, the modifications to the eigenvalues at first order in perturbation theory are

$$
E_{n}^{(1)}=\hbar \omega\left(n+\frac{1}{2}\right)+\alpha \frac{19 \hbar^{2} \omega^{2}}{192 m}\left(4 n^{2}+4 n+3\right)
$$

and

$$
E_{n}^{(2)}=\hbar \omega\left(n+\frac{1}{2}\right)-\alpha \frac{\hbar^{2} \omega^{2}}{4 m}\left(2 n^{2}+2 n+1\right) .
$$

Similarly, the modifications to the eigenvectors are

$$
\psi_{n}^{(1)}(x)=|n\rangle+\alpha \frac{19 \hbar \omega}{768 m}\left[A_{-4}|n-4\rangle-2 A_{-2}|n-2\rangle-2 A_{2}|n+2\rangle-A_{4}|n+4\rangle\right]
$$

and

$$
\psi_{n}^{(2)}(x)=|n\rangle-\alpha \frac{\hbar \omega}{16 m}\left[A_{-4}|n-4\rangle+A_{-2}|n-2\rangle+A_{2}|n+2\rangle+A_{4}|n+4\rangle\right],
$$

where $A^{\prime} s$ have been defined as

$$
\begin{aligned}
A_{-4} & =\sqrt{n(n-1)(n-2)(n-3)} \\
A_{-2} & =(n-2) \sqrt{(n-1)(n+1)}+(n-1) \sqrt{n(n-1)}+(2 n-1) \sqrt{(n-2)(n-1)} \\
A_{2} & =(n+1) \sqrt{n(n+2)}+(n+3) \sqrt{(n+1)(n+2)}+(2 n+6) \sqrt{n+2)(n+1)} \\
A_{4} & =\sqrt{(n+4)(n+3)(n+2)(n+1)}
\end{aligned}
$$

As one can see from (29) and (30), the quantum dynamics of the system (13) and (14) are quite different even a first order in perturbation theory and at first order in the parameter $\alpha$. 


\section{Conclusions and comments}

For a one-dimensional dissipative system with position depending coefficient, we have shown that it is possible to have two different Hamiltonians associated to the same classical dynamics, but they bring about completely different quantum dynamics. Since this happens for any potential $U(x)$ and due to the expression (13) and (14), this ambiguity occurs for a non numerable set of one-dimensional systems. In addition, one has implication on cahotic systems, since the classical equation of motion for the cahotic systems [12] may be formulated can be formulated using different nonequivalent time depending Hamiltonians (for one-dimensional cahotic motion), the behavior of the corresponding quantum system would have different types of quantum chaos. Finally, one needs to point out here that this ambiguity will appear also on the Hamiltonian formulation of classical and quantum statistical mechanics.

\section{Acknowledgments}

One of us, G. López, wants to thank V.I. Man'ko for his very useful discussions. 


\section{References}

1. J. Douglas, Trans. Amer. Math. Soc.,50 (1941) 71.

2. A. O. Caldeira and A.T. Legget, Physica A, 121 (1983) 587.

W.G. Unruh and W.H. Zurek, Phys. Rev. D, 40 (1989) 1071.

B.L. Hu, J.P. Paz and Y. Zhang, Phys. Rev. D, 45 (1992) 2843.

3. D. Darboux, Leçons sur la théorie général des surfaces et les applications géométriques du calcul infinitésimal, IViéme partie, Gauthoer-VIllars, Paris, 1984.

4. P. Havas, Act. Phys. Austr., 38 (1973) 145.

S. Okubo, Phys. Rev. D 22 (1980) 919.

V.V. Dodonov, V.I. Man'ko and V.D. Skarzhinsky, Hadr. Jour. 4 (1981) 1734.

G. Marmon, E.J. Salentan, A. Simoni and B. Vitale, Dynamical System:

a Differential Geometric Approach, Wiley, Chinchester, 1985.

R. Glauber and V.I. Man'ko, Sov. Phys. JEPT, 60 (1984) 450.

G. López, Intr. Jour. Theo. Phys.,37,5 (1998) 1617.

G. López, Rev. Mex. Fis., ]bf 45,6 (1999) 551.

5. G. López, Rev. Mex. Fis., 48,1 (2002) 10.

6. G. López, Ann. Phys., 251, 2(1996) 372.

7. P.G. Drazin, Nonlinear Dynamics, Cambridge University Press, 1992.

8. G. López, Partial Differential Equations of First Order and Their Applications to Physics, World Scientific, 1999.

9. F. John, Partial Differential Equations, Springer-Verlag, N.Y. 1974.

10. J.A. Kobussen, Act. Phys. Austr., 51 (1979) 193.

C. Leubner, Phys. Rev. A 86 (1987) 9.

C.C. Yan, Amer. J. Phys., 49 (1981) 296.

G. López, Ann. Phys., 251, 2(1996) 372.

11. A. Messiah, Quantum Mechanics Vol. II, John-Wiley and Sons, 1958.

12. G. Casati and B. Chirikov (Eds.), Quantum Chaos: between order and disorder, Cambridge University Press, 1995.

F. Benvenutto and G. Casati, Phys. Rev. Lett., 72 (1994) 1818.

E. Ott, Chaos in Dynamical Systems, Cambridge University Press, 1993.

G.M. Zaslavsky and N.N. Filonenko, Zh., Eksp. Teor.

Fiz. 65 (1973) 643.

K.N. Alekseev et al, Sov. Phys. Uspekhi, 35] (1992) 572.

R. Graham and M. Höhnerbach, Phys. Lett. A, 101,2 (1984) 61. 